\title{
SUPERVISED ENCODING FOR DISCRETE REPRESENTATION LEARNING
}

\author{
Cat P. Le $\quad$ Yi Zhou ${ }^{\star} \quad$ Jie Ding Vahid Tarokh $^{\ddagger}$ \\ * Department of Electrical and Computer Engineering, Duke University \\ $\dagger$ Department of Electrical and Computer Engineering, University of Utah \\ $\ddagger$ School of Statistics, University of Minnesota
}

\begin{abstract}
Classical supervised classification tasks search for a nonlinear mapping that maps each encoded feature directly to a probability mass over the labels. Such a learning framework typically lacks the intuition that encoded features from the same class tend to be similar and thus has little interpretability for the learned features. In this paper, we propose a novel supervised learning model named Supervised-Encoding Quantizer (SEQ). The SEQ applies a quantizer to cluster and classify the encoded features. We found that the quantizer provides an interpretable graph where each cluster in the graph represents a class of data samples that have a particular style. We also trained a decoder that can decode convex combinations of the encoded features from similar and different clusters and provide guidance on style transfer between sub-classes.
\end{abstract}

Index Terms - quantization, clustering, discrete representation, generative model

\section{INTRODUCTION}

Although deep learning has achieved great success in solving a variety of challenging tasks, most of the existing deep learning models do not provide sufficient interpretability for the learned features. For instance, classical supervised learning only maps the training samples to their corresponding labels, and often do not exploit the features of the samples. Consequently, such a learning framework typically produces a black-box model that provides little interpretability for the learned classification rule. As modern machine learning tasks involve increased model complexity and dimensionality, there is a rising interest in developing deep learning models that can provide a good visualization of the features and interpretable decision rules.

In this work, we combine a supervised-encoding technique with the $k$-means quantizer to build a supervised learning model with high classification accuracy and interpretability. Briefly speaking, we first train an encoder network that maps input data to low-dimensional embeddings with a softmax layer and the cross-entropy loss using the standard su-

This work was supported in part by Office of Naval Research Grant No. N00014-18-1-2244. pervised training. Such a pre-trained encoder ensures the encoded features of the training data samples to be linearly separable with regard to the labels. Then, the encoded features of the training data are clustered by a $k$-means quantizer, and each cluster is assigned a label by performing a majority vote. This quantization approach with supervised encoding enables the model to perform regular classification tasks and identify sub-classes of the data that correspond to different styles. Lastly, we also train a decoder that can reconstruct the data from its features and hence can generate new data samples with specified style mixtures.

Related Work. Applying quantization to the embedded features has been studied before, e.g. in [1-4], but it remains a challenge to achieve desired learning performance. In particular, some authors have suggested using a non-linear mapping to pre-process the data before performing the quantization, e.g. using an autoencoder for dimension reduction [1, 3, 5]. In [1], DEC model introduces an incorporated loss to jointly train an autoencoder and a quantizer. As a result, it improves the separation of data and makes the quantization process more efficient. The CAE- $l_{2}$ is another model proposed in [5] that consists of a convolutional autoencoder and a $k$-means quantizer. The model makes data features more separable in the Euclidean space by applying a $l_{2}$ normalization to the encoded features. Recently, [3] developed a DNM model that uses a self-organizing map [6,7] as the quantizer to map the embedded data into a predefined number of clusters. This approach can learn multiple different styles of the data within the same class, but it cannot perform classification tasks.

Our method is related to some prior work in image compression using deep neural networks. For example, a quantization method using soft assignments over time was proposed to obtain a hard clustering [4]. Compressive autoencoder [8] compresses lossy features using rounding-based quantization before entropy encoding. Our approach also connects to the literature of learning the distribution of data representations. In [2], GMVAE uses Gaussian mixture model on the latent space of VAE [9] to understand the distribution of latent representations. VQ-VAE is a model proposed in [10] that uses VQ as a bridge to connect the encoder and the decoder of VAE. These approaches learn the discrete latent distribution and can be considered as generative models. 


\section{SUPERVISED-ENCODING QUANTIZER}

Our supervised-encoding quantizer (SEQ) model consists of an encoder, a quantizer, and a decoder, as illustrated in Figure1 The SEQ training consists of the following steps, which are further elaborated in the following subsections.

1. Encoding: we pre-train the encoder by attaching a softmax layer to its output and train the encoder via standard supervised training;

2. Quantization: the encoded features produced by the pre-trained encoder are passed to the quantizer for clustering, as illustrated in Figure 2

3. Decoding: we further train a decoder that can reconstruct the original data samples from the encoded features.

Regarding the quantization step, various quantizers can be applied to the SEQ model, including $k$-means, vector quantization, self-organizing map, grow-when-required network [11], and Gaussian mixture model. For simplicity, we apply the $k$ means clustering algorithm [12] in the quantization step. We elaborate on each of the main steps as follows.

\subsection{Pre-training Encoder}

To obtain an interpretable embedding space, the encoder of the SEQ needs to be pre-trained with labeled data. First, we construct an encoder consisting of linear layers and/or convolutional layers. To train the encoder, we attach a Softmax layer to the output of the encoder. Then, we train such a neural network using the labeled data with cross-entropy loss and stochastic gradient descent. After the training, we remove the Softmax layer and keep the parameters of the encoder. In particular, the output embedding features $Z$ produced by the pretrained encoder are guaranteed to be linearly separable due to the supervised training with cross-entropy loss.

\section{2. $k$-means Quantization}

The pre-trained encoder maps each data sample $x$ to a corresponding embedded feature $Z(x)$. Then, as illustrated in Figure 2, the quantizer takes the embedded features $Z$ of all data samples as the input and applies the $k$-means clustering algorithm to quantize these features with a pre-defined number $K$. The clustering produces $K$ clusters with centers $C_{1}, C_{2}, \ldots, C_{K}$, and each of the features is assigned to the cluster that has the minimum distance between the feature and the cluster center. Such a clustering result naturally produces a topological graph, where each cluster consists of the features of samples that are close to each other in terms of the Euclidean distance. By choosing $K$ to be greater than the total number of classes, the $k$-means clustering can identify the sub-classes of the samples within each class of the data.

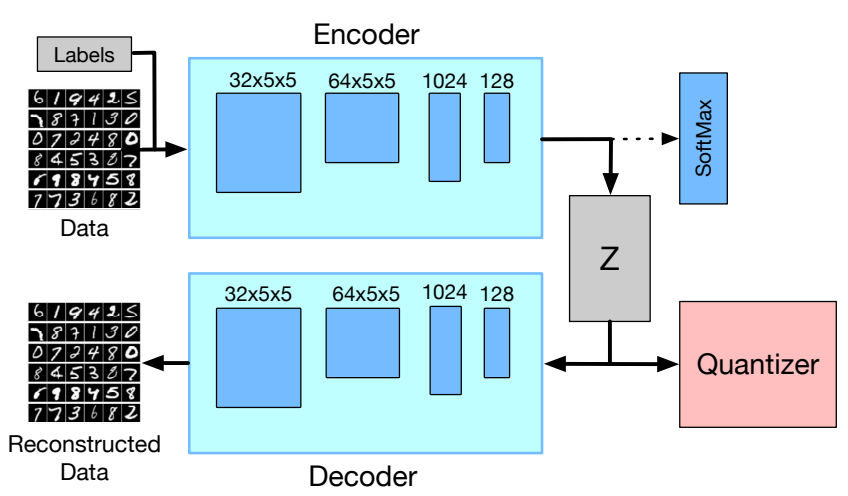

Fig. 1. Illustration of the proposed supervised-encoding quantizer (SEQ).

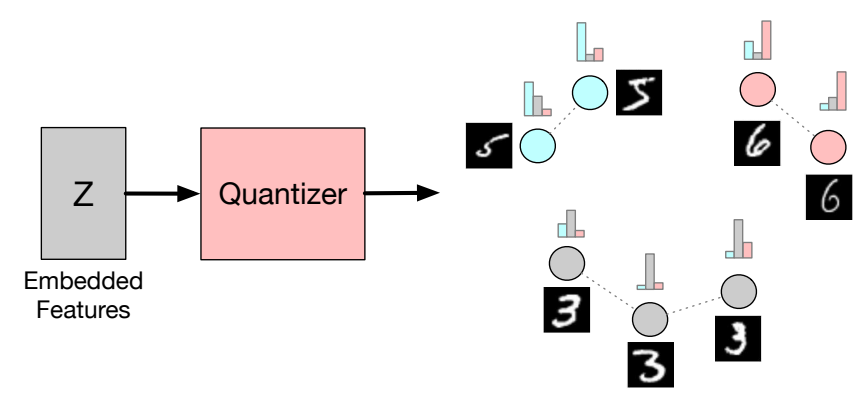

Fig. 2. Illustration of the quantization process.

To further perform a classification task, we use a simple approach based on the majority vote strategy, called histogrambased labeling. In particular, the label for each cluster is set to be the label with the highest frequency in that cluster.

In practice, we found that a larger $K$ usually leads to better classification performance but more computational costs. To tune an appropriate choice of the total number of clusters $K$ for the SEQ quantizer, we propose the following simple rule. We denote $P_{Q}$ as the classification accuracy of the quantizer, and $P_{E}$ as the classification accuracy of the encoder network achieved in the pre-training phase. Since the accuracy of the quantizer is upper bounded by that of the encoder, we search for the smallest value $K$ such that $P_{Q}>P_{E}-\epsilon$.

\subsection{Training Decoder}

We attach a decoder to the output of the encoder in order to reconstruct the data from the embedded features. First, we construct a decoder whose layers are symmetrical to the encoder's structure. To train the decoder, we fix all the parameters of the encoder and apply the MSE loss to measure the reconstruction error on the training data samples as:

$$
\mathcal{L}(\theta):=\left\|X-\mathcal{D}_{\theta}(\mathbf{s g}[\mathcal{E}(X)])\right\|^{2},
$$

where $\mathcal{E}$ denotes the pre-trained encoder that compresses the data samples $X$ into embedded features $Z$, sg denotes the 
stop-gradient operator which freezes the encoder's parameters, $\mathcal{D}_{\theta}$ denotes the decoder with the parameters $\theta$. We minimize the reconstruction loss $\mathcal{L}$ over the decoder parameters $\theta$ only using the stochastic gradient descent algorithm.

\section{EXPERIMENTAL STUDY}

\subsection{Performance Evaluation}

We evaluate the classification accuracy of SEQ on both the training dataset and the test dataset. In specific, we consider the following three network structures for the encoder network of the SEQ1:

1. LAE-2: the encoder has 2 fully connected hidden layers with dimensions dense(1024)-dense(128);

2. LAE-4: the encoder has 4 fully connected hidden layers with dimensions dense(1024)-dense(512)-dense(256)dense(128);

3. CAE-4: the encoder has 2 convolution layers and 2 fully connected hidden layers with dimensions $(32 \times$ $5 \times 5)$ - $(64 \times 5 \times 5)$-dense(1024)-dense(128).

In addition, regarding the $k$-means quantizer, we consider the total number of clusters $K$ ranging from 10 to 120 . We evaluate the performance of these SEQ models on the standard MNIST [13] and fashion-MNIST [14] datasets.

We first evaluate the clustering performance of the $k$ means quantizer of SEQ on the training dataset using unsupervised learning criteria. The metric for evaluating the clustering performance is defined by the percentage of training samples that are assigned to the correct label by the $k$-means quantizer as described in Section 2.2. We compare the clustering performance of SEQ with those of DEC [1], IDEC [15], DCEC [16], and CAE- $l_{2}$ [5], respectively. The results are shown in Table 1 The reported value in the parenthesis is the standard error of our average accuracy from re-samplings. Standard errors of other methods were not reported in previous works. The table shows that our SEQ achieves significantly higher accuracy on the training dataset compared with state-of-the-art methods. A possible reason is the advantage brought by the embedded space of SEQ that is well clustered in line with the true labels.

Next, we evaluate the classification performance of SEQ on the test dataset. In specific, for a given new test data sample, we pass it through the encoder to obtain its feature. Then, we identify the cluster with the closest center point to this feature vector and assign the corresponding cluster label to be the predicted label for the test data sample. Figure 3 shows the classification performance of SEQ on test data versus the number of clusters. We observe a notable improvement of the test accuracy as the number of clusters increases from 10

\footnotetext{
${ }^{1}$ Source code for the SEQ model is available at the GitHub link below: https://github.com/lephuoccat/Supervised-Encoding-Quantizer
}

Table 1. The clustering performance on MNIST

\begin{tabular}{ccccc}
\hline \hline DEC & IDEC & DCEC & $\begin{array}{c}\text { CAE- } l_{2} \\
k \text {-means }\end{array}$ & $\begin{array}{c}\text { SEQ } \\
k \text {-means }\end{array}$ \\
\hline 86.55 & 88.06 & 88.97 & 95.11 & $\mathbf{9 9 . 7 4 ( \mathbf { 0 . 0 4 6 ) }}$ \\
\hline \hline
\end{tabular}

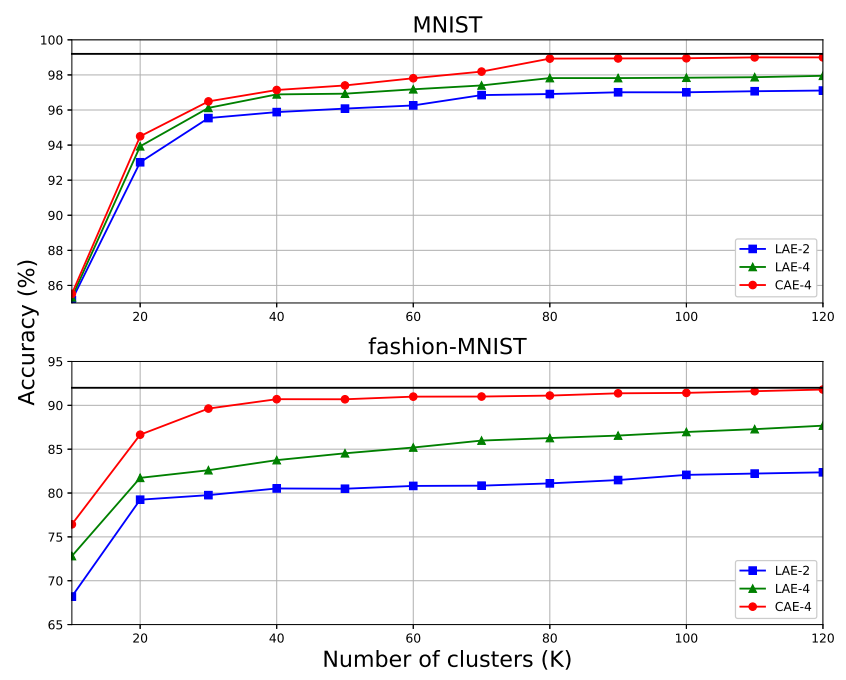

Fig. 3. The classification performance of SEQ with respect to the number of clusters for MNIST and fashion-MNIST.

to 120 . In particular, the maximum accuracy reaches $99 \%$ as $k \geq 100$ on the MNIST dataset. Also, the test performance improves when the number of layers in the encoder increases. Moreover, the SEQ model that adopts convolutional layers in the encoder outperforms the other models with linear layers in the encoder. Similar observations are made on the fashionMNIST dataset and the maximum accuracy is $91.8 \%$ when $k \geq 120$. The performance gap of the three SEQ models is more noticeable for fashion-MNIST, which is considered to be more challenging than MNIST. Also, the solid black horizontal line denotes the test accuracy of the pre-trained encoder, which upper-bounds the test performance of SEQ.

\subsection{Interpretability}

In this subsection, we show that the SEQ model can provide interpretable clustering results. In the top row of Figure 4 we present the images decoded from the average features of 50 cluster centers on the MNIST and fashion-MNIST datasets, respectively. Specifically, each decoded image is obtained by feeding the average of the features within each cluster to the trained decoder. As observed in the top two figures, the average of the features of the clusters in a certain class can be decoded into images with different styles. For example, for the MNIST dataset, the digit 1 and digit 7 have multiple different styles, which are captured by different clusters and are shown in the middle and bottom rows of Figure 4 (a). 


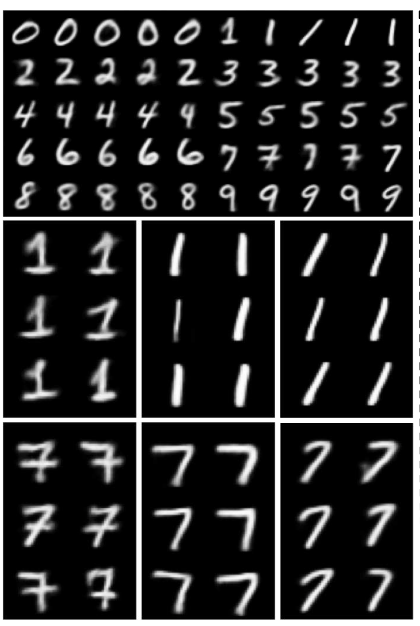

(a) MNIST

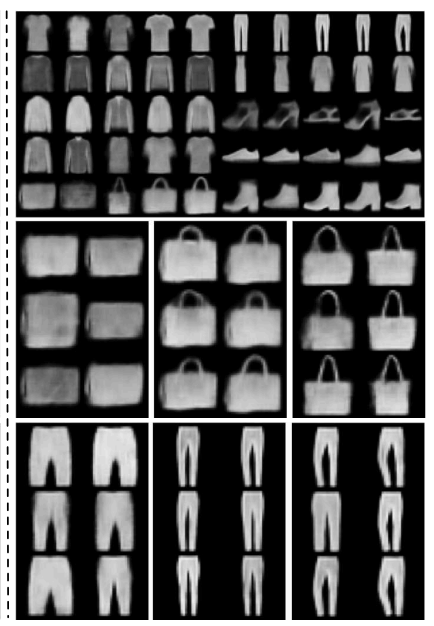

(b) fashion-MNIST

Fig. 4. Row 1: The average decoded images from the clustering points showing multiple styles for each class of data for MNIST and fashion-MNIST. Row 2-3: The mapped data samples from different clusters.

Similarly, for the fashion-MNIST dataset, the bag images have three different styles, i.e., no handle, short handle, and long handle, which are presented in the middle row of Figure 4 (b). The trousers also have three styles and are shown in the bottom row. The results indicate that the embedding space of the SEQ quantizer is interpretable in the sense that data samples with the same style are clustered together.

\subsection{Semi-generative Model}

Given that the SEQ model is capable of clustering similar data into a sub-classes, we consider the output of this model as a graph network where each node of the graph is the center clustering point. We also demonstrate that the embedding space of the SEQ model is smooth and continuous in decoding, in the sense that we can combine and interpolate the feature vectors within the same cluster and obtain decoded images with the same style.

We consider convex combinations of three features within a certain cluster in the form of $x=\sum_{i=1}^{3} \alpha_{i} x_{i}$, where we vary $\alpha_{1}, \alpha_{2} \in(0,0.5)$ and $\alpha_{3}=1-\alpha_{1}-\alpha_{2}$. For any such convex combination, the newly generated feature is considered to belong to the same class. In Figure 5(a), we present the new data samples that are decoded from the features using convex combinations of three features with different combination coefficients. In each block of Figure 5(a), the decoded images of the three selected features (denotes as $x_{1}, x_{2}, x_{3}$ ) from the same cluster are located at the top left, top right and bottom right, respectively, and are highlighted by the white boxes. The decoded images from the convex combined features are shown in the blocks. We observe that the generated images have clear shapes and similar structures.

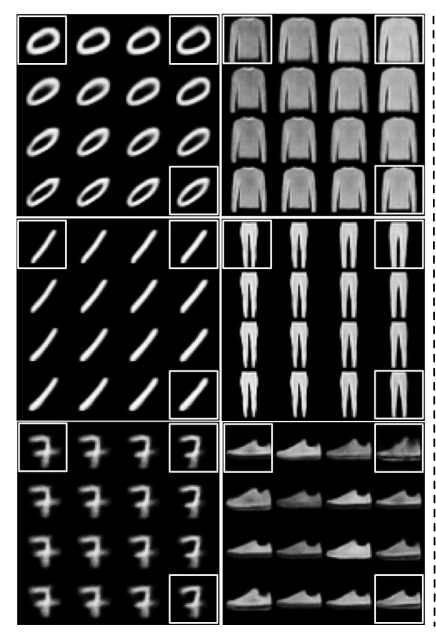

(a) Similar style

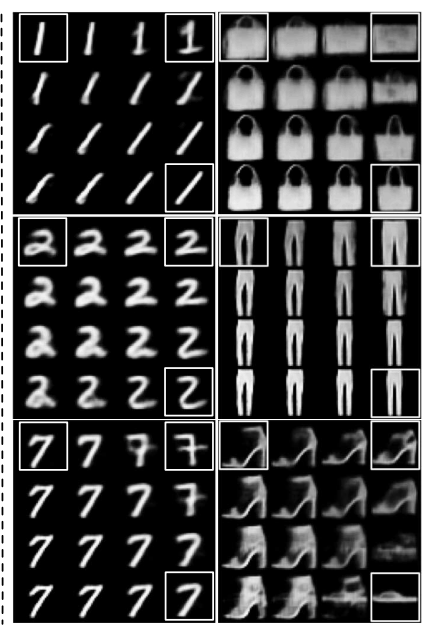

(b) Different styles
Fig. 5. The generative data using convex hull of three data samples from (a) the same cluster and (b) different clusters for MNIST and fashion-MNIST. The images inside the white boxes are the original data samples.

Lastly, we applied convex combinations to three data samples from different clusters (styles) within the same class. As shown in Figure 5(b), the generated images are smooth transitions between different styles. Interestingly, the generated images also have clear shapes and resemble the class structures. Additionally, they do not suffer from any blurring artifact, which can be an issue in other generative models such as GANs [17] and VQ-VAE [10].

\section{CONCLUSION}

We provide a new perspective of looking at supervised classification and unsupervised generative models with nonlinear feature mapping. We first propose a supervised learning model SEQ that applies a quantizer to cluster and classify the encoded features. We surprisingly found that each quantizer provides an interpretable representation of a set of images that have a particular style. As a result, the encoder can be regarded as an interpretable dimension reduction that compresses data in such a manner that closer styles in the data domain correspond to closer features in the feature domain. From the computation viewpoint, the new supervised learning method requires low-complexity layers for the encoder and decoder which can enable faster training. We also show how to generate data using the convex hull from a few data samples in the network model. Since the model learns to cluster the data into sub-classes that have different styles, it is able to precisely control the generating process to produce the desired data style. As an ongoing work, we are applying the developed techniques in medical applications, e.g. generating specific types of cancer images. 


\section{REFERENCES}

[1] Junyuan Xie, Ross Girshick, and Ali Farhadi, "Unsupervised deep embedding for clustering analysis," in Proc. International conference on machine learning (ICML), 2016, pp. 478-487.

[2] Nat Dilokthanakul, Pedro AM Mediano, Marta Garnelo, Matthew CH Lee, Hugh Salimbeni, Kai Arulkumaran, and Murray Shanahan, "Deep unsupervised clustering with gaussian mixture variational autoencoders," arXiv preprint arXiv:1611.02648, 2016.

[3] Mehran Pesteie, Purang Abolmaesumi, and Robert Rohling, "Deep neural maps," arXiv 1810.07291, 2018.

[4] Eirikur Agustsson, Fabian Mentzer, Michael Tschannen, Lukas Cavigelli, Radu Timofte, Luca Benini, and Luc Van Gool, "Soft-to-hard vector quantization for end-to-end learned compression of images and neural networks," arXiv : 1704.00648, vol. 3, 2017.

[5] Caglar Aytekin, Xingyang Ni, Francesco Cricri, and Emre Aksu, "Clustering and unsupervised anomaly detection with $\ell_{2}$ normalized deep auto-encoder representations," in Proc. International Joint Conference on Neural Networks (IJCNN), 2018, pp. 1-6.

[6] Teuvo Kohonen, "The self-organizing map," Proceedings of the IEEE, vol. 78, no. 9, pp. 1464-1480, 1990.

[7] Teuvo Kohonen, Erkki Oja, Olli Simula, Ari Visa, and Jari Kangas, "Engineering applications of the selforganizing map," Proceedings of the IEEE, vol. 84, no. 10, pp. 1358-1384, 1996.

[8] Lucas Theis, Wenzhe Shi, Andrew Cunningham, and Ferenc Huszár, "Lossy image compression with compressive autoencoders," arXiv :1703.00395, 2017.

[9] Diederik P Kingma and Max Welling, "Auto-encoding variational bayes," arXiv :1312.6114, 2013.
[10] Aaron van den Oord, Oriol Vinyals, et al., "Neural discrete representation learning," in Proc. Advances in Neural Information Processing Systems (NeurIPS), 2017, pp. 6306-6315.

[11] Stephen Marsland, Jonathan Shapiro, and Ulrich Nehmzow, "A self-organising network that grows when required," Neural Networks, vol. 15, no. 8-9, pp. 10411058, 2002.

[12] Edward W Forgy, "Cluster analysis of multivariate data: efficiency versus interpretability of classifications," Biometrics, vol. 21, pp. 768-769, 1965.

[13] Yann LeCun, Corinna Cortes, and CJ Burges, "Mnist handwritten digit database," AT\&T Labs [Online]. Available: http://yann. lecun. com/exdb/mnist, vol. 2, pp. 18, 2010.

[14] Han Xiao, Kashif Rasul, and Roland Vollgraf, "Fashionmnist: a novel image dataset for benchmarking machine learning algorithms," arXiv :1708.07747, 2017.

[15] Xifeng Guo, Long Gao, Xinwang Liu, and Jianping Yin, "Improved deep embedded clustering with local structure preservation.," in Proc. International Joint Conference on Artificial Intelligence (IJCAI), 2017, pp. 17531759.

[16] Xifeng Guo, Xinwang Liu, En Zhu, and Jianping Yin, "Deep clustering with convolutional autoencoders," in Proc. International Conference on Neural Information Processing, 2017, pp. 373-382.

[17] Ian Goodfellow, Jean Pouget-Abadie, Mehdi Mirza, Bing Xu, David Warde-Farley, Sherjil Ozair, Aaron Courville, and Yoshua Bengio, "Generative adversarial nets," in Proc. Advances in neural information processing systems (NeurIPS), 2014, pp. 2672-2680. 